Rev, Elev. Méd. vét. Pays trop., 1978, 31 (4) : 411-415.

\title{
A propos d'un nouveau cas de botulisme hydrique de type D survenu au Sénégal. Considérations étiopathogéniques
}

\author{
par M. P. DOUTRE $(*)$ et B. TOURE $(* *)$
}

\begin{abstract}
RÉSUMÉ
Un nouveau foyer de botulisme hydrique de type $D$, dû à la présence du cadavre d'un chat dans un puits, est apparu au Sénégal, dans la région de Diourbel, provoquant la mort de 7 chevaux, 2 ânes, 5 moutons et 1 chèvre. La souche en cause est isolée du foie d'un cheval. Dans la discussion, les auteurs insistent sur le rôle que doit jouer la sécheresse dans la répétition des cas de botulisme hydrique en zone d'élevage extensif sahélo-soudanienne. Un schéma général étiopathogénique est proposé.
\end{abstract}

Au cours de la seconde quinzaine de mars 1978, 7 chevaux, 2 ânes, 5 moutons et 1 chèvre meurent après avoir absorbé l'eau d'un puits d'un village du Baol (Keur Ndiouga Mbaye, arrondissement de Ndindy, région de Diourbel), d'où est retiré le cadavre d'un chat. La symptomatologie présentée par les animaux avant leur mort (paralysies flasques) et les commémoratifs font suspecter immédiatement un foyer du botulisme hydrique. Au Sénégal (7), comme au Tchad (10), des observations similaires ont déjà été rapportées.

La présente note se propose de signaler dans quelles circonstances a été isolée la seconde souche de Clostridium botulinum type $\mathrm{D}$ au Sénégal et d'émettre des hypothèses concernant la relation possible entre les cas de botulisme hydrique rencontrés ces dernières années et l'épizootie survenue, à partir des années 60, dans la zone d'élevage extensif du Ferlo, et

(*) Chef du Service de Bactériologie. Laboratoire national de l'Elevage et de Recherches vétérinaires (I. S. R. A.) B. P. 2057, Dakar-Hann, Sénégal.

(**) Technicien supérieur. Laboratoire national de l'Elevage et de Recherches vétérinaires (I. S. R. A.). l'origine éventuelle, liée à la sécheresse, de la fréquence des noyades de petits mammifères (le plus souvent des carnivores) dans cette région géographique du pays.

\section{MATÉRIEL ET MÉTHODES}

\section{1) MATÉRIEL}

Au niveau du foyer, de l'eau du puits a été récoltée, avant le curage, par un agent de la Santé et de la Production animales. Ce prélèvement est remis aux représentants du Laboratoire.

Un seul malade, un cheval, qui ne peut se lever qu'avec l'aide du propriétaire, est observé mais non sacrifié, car une éventuelle guérison peut être envisagée. Par contre, un cadavre, vieux de $24 \mathrm{~h}$, appartenant à la même espèce animale est exhumé et un fragment de foie prélevé.

\section{2) MÉTHODES}

- Inoculation à la souris, par voie intrapéritonéale, de $1 \mathrm{ml}$ d'eau de puits ; 
- Ensemencement en bouillon VF, glucosé à 1 p. 100 de l'eau de puits, de fragments de foie chauffé $10 \mathrm{mn}$ à $80^{\circ} \mathrm{C}$ et non chauffé, culture 5 jours à $37^{\circ} \mathrm{C}$, recherche de la présence éventuelle d'une toxine dans le surnageant de ces cultures mixtes ;

- Séroneutralisation : lorsqu'une toxine est mise en évidence, la séroneutralisation sur souris est réalisée, selon la méthode classique, en mettant en présence $100 \mathrm{DMM}$ /souris de toxine (sous un volume de $0,1 \mathrm{ml}$ ) et 1 unité antitoxique de chacun des sérums antitoxiques $\mathbf{A}, \mathbf{B}$, C, D, E fournis par l'Institut Pasteur de Paris (sous le même volume). La mortalité des souris est notée pendant 3 jours ;

- Isolement de la souche en cause sur milieux solides, à la fois, en milieu VF, gélosé et glucosé, réparti en tubes de Veillon, et sur boîtes de Pétri (gélose au sang, extraits de viande et de levure, cystéine (I)). L'anaérobiose est réalisée avec le système Gas Pak (BD Mérieux).

\section{RÉSULTATS}

- L'inoculation de $1 \mathrm{ml}$ d'eau de puits aux souris par voie intrapéritonéale provoque leur mort en $48 \mathrm{~h}$.

- Le surnageant de la culture mixte de l'eau de puits se révèle atoxique pour la souris. L'eau de puits rapportée ne contenait donc que de la toxine ;

- Les surnageants des cultures mixtes des fragments de foie provoquent la mort des souris à la dose de $0,1 \mathrm{ml}$ de la dilution $10^{-5}$;

- La séroneutralisation réalisée avec l'un de ces surnageants (culture de foie chauffé) montre que l'on se trouve en présence du type D de Cl. botulinum;

- La souche en cause est isolée sur boîte de Pétri sans difficulté. Ses caractères biochimiques sont identiques à ceux de la première souche isolée (3). Toutefois, la quantité de toxine convulsivante, non antigénique, produite en bouillon VF glucosé, est suffisamment faible pour ne pas interférer dans le sérotypage (surnageant d'une culture mixte, dilué à $100 \mathrm{DMM} /$ souris, sous un volume de $0,1 \mathrm{ml}+1$ unité antitoxique de chacun des sérums anti, sous le même volume), ce qui n'était pas le cas avec la première souche isolée (3) ;

- La DMM/souris de la toxine de culture préparée avec la présente souche se situe entre $10^{-6}$ et $10^{-7} \mathrm{ml}$ (souris de $20 \mathrm{~g}$ inoculées par voie intra-péritonéale).

\section{DISCUSSION}

L'apparition d'un nouveau foyer de botulisme de type $\mathrm{D}$, d'origine hydrique, est ainsi démontrée bactériologiquement.

Une première remarque vient à l'esprit. Les habitants du village ont consommé, en même temps que les futures victimes animales, l'eau de l'unique puits et n'ont cependant présenté aucun signe de la maladie. Bien que le botulisme de type D soit connu chez l'homme (4), il est fort possible que deux facteurs puissent expliquer l'absence de symptôme chez les villageois : une certaine hyposensibilité d'espèce face au type $\mathrm{D}$ de $\mathrm{Cl}$. botulinum (l'homme étant beaucoup plus sensible aux types A, B et $E$ ) et le fait que la quantité d'eau ingérée, donc de toxine par chaque individu, ait été relativement faible. En outre, dès les premières mortalités animales, le puits fut plusieurs fois curé et les restes du chat éliminés. Ces interventions ont certainement contribué à diminuer la toxicité de l'eau. Le foyer du Kanem (Tchad), de type $C$, avait suscité les mêmes réflexions de la part de PROVOST (10).

En 1971, lors des VII ${ }^{c}$ Journées Médicales, tenues à Dakar, nous avions proposé un schéma étiopathogénique dı botulisme des herbivores vivant dans le Ferlo, en considérant le rôle possible joué par le botulisme d'origine hydrique et en émettant l'hypothèse que « dans un ou plusieurs forages (les bassins-réservoirs sont entièrement à l'air libre, de même que les abreuvoirs) ou que dans un des anciens puits, l'eau souillée par un cadavre de chat ou de petit mammifère quelconque, ait été la cause d'une intoxication botulique limitée. Ensuite, les restes de ces animaux morts, disséminés dans un rayon de plusieurs centaines de mètres autour du point d'eau contaminé, auraient été à l'origine de la propagation des spores botuliques; leur absorption étant facilitée par l'ostéophagie présentée par des bovins en état permanent d'aphosphorose. La dissémination des restes, des ossements des nouvelles victimes et leur consommation par des animaux carencés auraient permis à la maladie de faire tache d'huile et de s'étendre progressivement à toute la région d'élevage $»(9)$. Le schéma étiopathogénique suivant avait été proposé : 


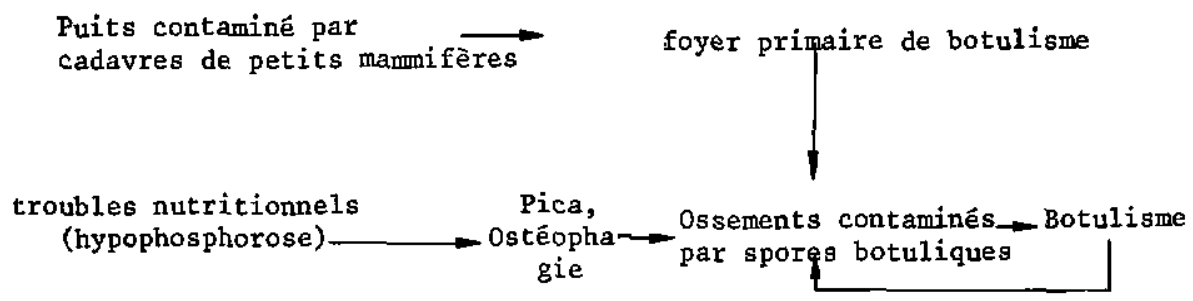

Avec le temps et l'acquisition d'une meilleure connaissance de l'influence des variations des conditions climatiques sur la vie animale de la partie septentrionale du Sénégal, il est apparu que ce schéma pouvait être complété et que les contaminations répétées de certains puits pouvaient trouver une explication dans le déficit pluviométrique noté depuis 12 ans dans les zones sahélienne et soudano-sahélienne. En effet, on remarque que :

- tous les foyers de botulisme hydrique observés se localisent au nord (région sylvopastorale ou arachidière) (7), bien que la presque totalité des puits soit pourvue d'une margelle cimentée, surélevée d'un mètre environ;

- dans le sud du pays (Casamance), les puits, très nombreux, sont le plus souvent entourés de quelques troncs d'arbres, disposés au carré, sur lesquels s'appuie l'exhaure manuelle. Ce dispositif, haut d'une trentaine de centimètres, n'assure qu'une protection très imparfaite de l'orifice et les risques de chute de petits mammifères devraient être accrus (Haute-Casamance en particulier). Or le botulisme est totalement inconnu dans toute la partie méridionale du Sénégal.

Dans la partie septentrionale, les espèces animales responsables des cas de botulisme hydrique sont essentiellement les petits carnivores prédateurs. En tout premier lieu, vient le chat haret, puis les viverridés (genettes, civettes, mangoustes) auxquels on peut joindre un mustellidé (le zorille). Un rongeur sciuridé (l'écureuil fouisseur dit improprement « rat palmiste "), qui s'alimente au détriment des plantes cultivées, a également été rencontré en décomposition dans l'eau de curage d'un puits (7). L'installation progressive et discontinue de la sécheresse a probablement réduit le nombre des carnivores par raréfaction des proies (rongeurs, oiseaux). Dans la lutte pour leur survie, certains individus se sont rapprochés des communautés humaines, à la fois pour s'alimenter et s'abreuver. Les espèces qui viennent d'être énumérées, essentieliement nocturnes (sauf l'écureuil fouisseur), peuvent être attirées par l'humidité du sol qui entoure le puits (ou la céane en Mauritanie), d'où, pendant la journée, l'eau est remontée d'une façon presque continue. De nuit, lorsque toute flaque a disparu, un animal assoiffé peut, dans sa quête, franchir la margelle, lorsqu'elle existe, et tomber accidentellement dans le puits.

L'action insidieuse de la "désertification 》 se manifesterait là comme dans d'autres domaines, par exemple la disparition progressive d'espèces végétales arborées (mort et absence de régénération) ou l'abaissement de la limite méridionale de l'aire de répartition d'une avifaune sud-saharienne ( 2 espèces de la famille des Alaudidés, particulièrement déserticole : Eremopterix nigriceps (alouette-moineau à front blanc, devenue résidente et nicheuse dans le Ferlo) et Alaemon alaudipes (sirli du désert).

Les variations pluviométriques ont interféré, d'une autre façon, lors de l'hivernage non déficitaire de 1975. Cette année, dès la fin des précipitations, la moitié nord du Sénégal a vu soudain apparaître une prolifération exceptionnelle, brutale et catastrophique pour les cultures de rongeurs (en particulier Arvicanthis niloticus). Le phénomène intéressa même les Léporidés. Dans la partie méridionale du pays (Casamance), aux conditions climatiques plus stables, rien de semblable n'était enregistré. Ce genre d'explosion des populations de rongeurs, bien connu dans d'autres parties du monde, peut s'expliquer à la fois par l'existence de conditions trophiques particulièrement favorables et par l'absence ou le relâchement de la prédation. A mesure que la saison sèche progressalt, dès les premiers mois de 1976, tout rentrait progressivement dans l'ordre et les rongeurs voyaient leur densité décroître mais en zone sylvo-pastorale, on assistait à une nouvelle flambée de botulisme. Ces derniers cas étant liés à la consommation directe par les bovins de cadavres de rats (rencontrés dans le rumen de zébus autopsiés au forage de Tatki, 1976).

Ces différentes considérations nous amènent à compléter le schéma étiopathogénique précédent de la façon suivante : 


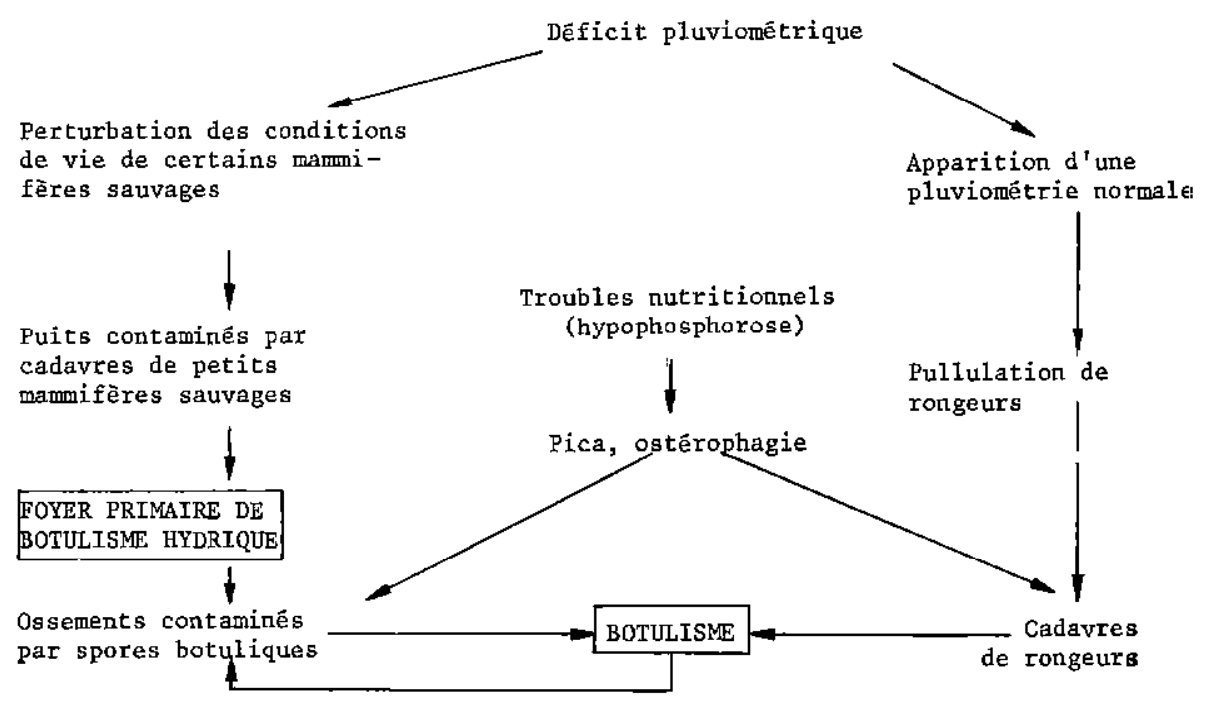

REMERCIEMENTS

Nous tenons à remercier Monsieur DIA, Chef du Secteur de Diourbel (Santé et Production animales) de nous avoir permis d'étudier ce nouveau foyer de botulisme hydrique.

\title{
SUMMARY
}

On a new outbreak of hydrous botulism type D

in Senegal. Etiopathogenic considerations

\begin{abstract}
A new outbreak of botulism type $\mathrm{D}$, caused by the absorption of water contaminated by the corpse of a drowned cat, is reported from the Diourbel area in Senegal. 7 horses, 2 donkeys, 5 sheep and 1 goat died. The strain involved was isolated from the liver of a dead horse. Along the discussion, the authors emphasize on the part played by drought in the repetition of cases of hydrous botulism in the sahelo-sudanian extensive cattle breeding zone. A general etiopathogenic scheme is proposed.
\end{abstract}

\section{RESUMEN}

A propósito de un nuevo caso de botulísmo hídrico de typo $D$ ocurrido en Senegal. Consideraciones etiopatogenicas

Un nuevo foco de botulismo hídrico de tipo $\mathrm{D}$, causado por la presencia del cadáver de un gato en un pozo, ocurró en Senegal, en la región de Diourbel, provocando la muerte de 7 caballos, 2 asnos, 5 ovejas y 1 cabra. Se aisló la cepa causadora del hígado del caballo. En la discusión, los autores insisten en el papel que debe desempeñar la sequia en la repetición de los casos de botulismo hídrico en zona de ganaderia extensiva sahelo-sudanesa. Se propone un plan general etiopathogénico.

\section{BIBLIOGRAPHIE}

1. BUTTIAUX (R. H.), TACQUET (A.). Manuel de techniques bactériologiques, Paris, Editions médicales Flammarion, 1969 et éd. ultérieures.

2. CALVET (H.), PICART (P.), DOUTRE (M. P.), CHAMBRON (J.). Aphosphorose et botulisme au Sénégal. Rev. Elev. Méd. vét. Pays trop., 1965, 18 (3) : 249-282.
3. CHAMBRON (J.), MARTEL (J. L.), DOUTRE (M. P.). Le botulisme équin au Sénégal. Premier isolement de Clostridium botulinum type D. Rev. Elev. Méd. vét. Pays trop., 1971, 24 (I) : 1-7.

4. DEMARCHI (J.), MOURGUES (C.), ORIO (J.), PREVOT (A. R.). Existence du botulisme humain de type D. Bull. Acad. nat. Méd., 1958, 142 : 580-582. 
5. DOUTRE (M. P.). Première observation du botulisme $\mathrm{C}$ beta chez le porc au Sénégal. Rev. Elev. Méd. vét. Pays trop., 1967, 20 (2) : 351-353.

6. DOUTRE (M. P.). Botulisme de type $C$ chez une tourterelle (Streptopelia roseogrisea bornuensis) du Ferlo (Sénégal). Rev. Elev. Méd. vét. Pays trop., 1967, 20 (4) : 601-604.

7. DOUTRE (M. P.). Fréquence au Sénégal du botulisme animal d'origine hydrique. Rev. Elev. Méd. vét. Pays trop., 1969, 22 (I) : 29-31.

8. DOUTRE (M. P.), CHAMBRON (J.). Le botulisme des ruminants et des équidés au Sénégal. Rev. Elev. Méd. vét. Pays trop., 1966, 19 (4) : 495-510.

9. DOUTRE (M. P.), CHAMBRON (J.). Le botulisme des ruminants et des équidés au Sénégal et en Mauritanie. Conséquence pathologique des troubles nutritionnels. VII ${ }^{\mathrm{e}}$ Journées médicales, Dakar, 11-16 janvier $1971,12 \mathrm{p}$.

10. PROVOST (A.), HAAS (P.), DEMBELLE (M.). Premiers cas au Tchad de botulisme animal (type C) : intoxication de dromadaires par l'eau d'un puits. Rev. Elev. Méd. vét. Pays trop., 1975, 28 (I) : 9-11. 